\title{
EFECTO DEL USO DEL SUELO SOBRE LAS FORMAS DE FÓSFORO DE UN ANDISOL
}

\author{
Carlos Henríquez, ${ }^{1 / *}$ \\ Palabras clave: Fraccionamiento de P; disponibilidad de P; manejo del suelo y P; metodología de Hedley. \\ Keywords: P fractionation; availability of P; soil management and P; Hedley methodology.
}

Recibido: $13 / 04 / 15$

\section{RESUMEN}

Se evaluó cómo el uso agrícola del suelo afecta las formas de P en un Typic Hapludand. En Juan Viñas, Costa Rica, se eligió un área de 8,4 ha compuesta de 3 lotes adyacentes y dedicados, por más de 20 años, a bosque secundario y a los cultivos de caña de azúcar y café. Se tomaron muestras de suelo por medio de un diseño de cuadrícula de 8x6 puntos de muestreo, distanciados a $40 \mathrm{~m}$. A las muestras de suelo se les aplicó la metodología de fraccionamiento de $\mathrm{P}$ propuesta por Hedley et ál. (1982). El P total varió entre 936 y $2516 \mathrm{mg} \cdot \mathrm{kg}^{-1}$. En los 2 manejos agrícolas, caña de azúcar y café, esta variable fue estadísticamente similar (1934 y 1754 mg.kg ${ }^{-1}$ respectivamente) y ambas fueron mayores al área de bosque (1419 $\mathrm{mg} \cdot \mathrm{kg}^{-1}$ ) lo que sugiriere un balance total positivo del P, debido muy posiblemente a la fertilización acumulada a través de los años. El área de cultivo del café mostró los mayores valores de P-lábil y $\mathrm{P}$-sesquióxidos-Fe/Al, relacionado posiblemente a la mayor fertilización en comparación con las áreas de caña de azúcar y bosque. El mayor valor de P-residual se encontró en el cultivo de caña de azúcar, con casi $48 \%$ del P total. El P orgánico constituyó entre el 27 y el $29 \%$ del total de P, lo que constituye también un reservorio importante.

Aceptado: 15/07/15

\begin{abstract}
Effect of land-use on the $P$ forms of an Andisol. The effect of agricultural land use on the forms of $\mathrm{P}$ in a Typic Hapludand was evaluated. An area of 8.4 ha composed of 3 adjacent lots, dedicated for over 20 years to secondary forest and plantings of sugarcane and coffee, was chosen at Juan Viñas, Costa Rica. Soil samples were taken using an $8 \times 6$ grid layout of sampling points, spaced at $40 \mathrm{~m}$. Soil samples were analyzed with the $\mathrm{P}$ fractionation methodology proposed by Hedley et ál. (1982). Total $\mathrm{P}$ varied between 936 and $2516 \mathrm{mg} \cdot \mathrm{kg}^{-1}$. Both coffee and sugar cane lots (1934 y 1754 mg. $\mathrm{kg}^{-1}$ respectively), showed the highest values of total-P compared with the forest (1419 mg.kg${ }^{1}$ ), suggesting a positive overall balance of $\mathrm{P}$ due to its accumulation through the years. The coffee area showed the highest values of P-labile and $\mathrm{P}$-sesquioxides-Fe/Al, which was related to usual higher fertilization compared with areas of sugarcane and forest. The greatest value of P-residual was found in sugarcane with almost $48 \%$ of total P. The P-organic varied 27 to $29 \%$ of the total suggesting its importance as valuable sink of P. We conclude that the type of land use had a significant effect on the forms of $\mathrm{P}$ which
\end{abstract}

1 Autor para correspondencia. Correo electrónico: carlos.henriquez@ucr.ac.cr

Centro de Investigaciones Agronómicas, Universidad de Costa Rica. 
Se concluye que el tipo de uso del suelo tuvo un efecto importante sobre las formas de P, lo cual se observó tanto en la cantidad como en la distribución de las diferentes formas de $\mathrm{P}$ en el suelo. was observed in both the quantity and distribution of different forms of $\mathrm{P}$ in soil.

\section{INTRODUCCIÓN}

El contenido y el comportamiento del fósforo $(\mathrm{P})$ en los suelos para uso agrícola, está determinado inicialmente por las propiedades originales del material parental, el tipo de arcilla dominante, por la fracción orgánica, así como por otras propiedades y procesos de naturaleza biológica y química (Fassbender y Bornemisza 1987, Condron y Newman 2011). Asimismo, el manejo agronómico al que ha sido sometido el suelo, puede provocar variaciones importantes del $\mathrm{P}$ que modifican igualmente su dinámica en el suelo (Hedley et ál. 1994, Neufeldt 2000, Meason 2009, Linquist et ál. 2011, Borda et ál. 2014). Es un hecho de que las reacciones del P, así como la de otros elementos en el suelo, son complejas y su disponibilidad real para las plantas es afectada por una gran diversidad de procesos a veces muy complejos, por la diversidad de componentes e interacciones que participan (Olsen y Khasawneh 1980, Frossard et ál. 2000, Condron y Newman 2011).

En el suelo, el P total está constituido por varias formas o fracciones, las cuales han sido definidas principalmente por su relación con otros componentes tanto minerales como orgánicos (Olsen y Khasawneh 1980, Beck y Sánchez 1994). Es así como diversas metodologías de fraccionamiento de las formas de $\mathrm{P}$ han sido propuestas tanto en suelos de zonas templadas como tropicales (Condron y Newman 2011). Todas ellas se basan en la extracción secuencial de una determinada muestra de suelo con diferentes soluciones las cuales disuelven correspondientemente ciertos componentes ligados con el $\mathrm{P}$, los cuales son disueltos para su posterior determinación (Olsen y Khasawneh 1980). De esta manera se han identificado diferentes categorías como $\mathrm{P}$ lábil, $\mathrm{P}$ ligado a sesquióxidos de $\mathrm{Fe} / \mathrm{Al}, \mathrm{P}$ ligado a fosfatos de calcio, $\mathrm{P}$ ligado a formas orgánicas $\mathrm{y}$ finalmente una fracción denominada como $\mathrm{P}$ residual u ocluido el cual está formado estructuras más complejas en el suelo que tienen muy baja disponibilidad para las plantas (Fassbender y Bornemisza 1987). Hedley et ál. (1982) propusieron una metodología que ha mostrado una muy buena relación con el crecimiento de los cultivos (Magid 1993, Beck y Sánchez 1994, Henríquez y Killorn 2005).

Se ha propuesto que la disponibilidad de $\mathrm{P}$ para las plantas depende en mucho de la interacción entre sus diferentes formas o fracciones en el suelo en términos de los procesos que determinan el paso de las formas poco a muy disponibles para los cultivos y viceversa; esto ocurre en mucho mediante procesos de sorción y desorción en el suelo, aunque también los procesos que tienen que ver con la fracción orgánica del elemento están involucrados, como por ejemplo la mineralización y la formación de complejos (Soto 1998, Ball et ál. 1993, Henríquez 2005). Estos procesos, pueden ser modificados en una forma importante y como se dijo anteriormente, por el manejo o uso agronómico al que el suelo es sometido.

Este estudio tuvo como objetivo estudiar el efecto del uso del suelo con respecto a la distribución de las formas de $\mathrm{P}$ en el suelo y su relación con la disponibilidad del suelo.

\section{MATERIALES Y MÉTODOS}

El lugar del estudio se ubicó en la localidad de Juan Viñas, Costa Rica, sobre un Typic Hapludand. Se eligió una área de aproximadamente 8,4 ha, en la cual estaban ubicados 3 lotes contiguos, 
los cuales habían estado dedicados por más de 20 años uno a bosque secundario y los otros 2 a los cultivos de caña de azúcar y del café. En el área elegida para el estudio se llevó a cabo un muestreo de suelos en forma de cuadrícula regular de 6x8 puntos de muestreo distanciados $40 \mathrm{~m}$ entre sí. En cada uno de estos puntos o centroides, se tomaron 4 muestras de suelo alrededor con el fin de conformar una muestra compuesta para su posterior análisis. La descripción detallada del área de estudio, así como otros datos de caracterización, fueron presentados por Henríquez et ál. (2005) al hacer un estudio geoestadístico de la variabilidad espacial de las propiedades de fertilidad del suelo de área. Algunos datos del análisis químico, se presentan en el Cuadro 1.

Cuadro 1. Algunas propiedades químicas del suelo $(0-20 \mathrm{~cm})$ en 3 sistemas de manejo agronómico de un Typic Hapludand. Juan Viñas, Costa Rica (Henríquez et ál. 2005).

\begin{tabular}{|c|c|c|c|c|c|c|c|}
\hline Áreas & $\begin{array}{c}\mathrm{P} \\
\mathrm{mg} \cdot \mathrm{kg}^{-1}\end{array}$ & $\mathrm{pH} \mathrm{H} \mathrm{H}_{2} 0$ & \multicolumn{4}{|c|}{$\operatorname{cmol}(+) \cdot \mathrm{kg}^{-1}$} & $\begin{array}{c}\text { M.O. } \\
\%\end{array}$ \\
\hline Caña azúcar & 5,2 & 5,5 & 1,7 & 0,42 & 0,15 & 0,7 & 11,72 \\
\hline Café & 11,6 & 5,4 & 7,2 & 1,49 & 0,46 & 1,7 & 6,96 \\
\hline Bosque & 3,9 & 5,4 & 8,4 & 3,04 & 0,27 & 1,1 & 7,40 \\
\hline
\end{tabular}

Seguidamente y una vez obtenidas las muestras de suelo de cada punto, estas fueron llevadas al laboratorio, luego secadas y pasadas por una malla de $2 \mathrm{~mm}$ y analizadas de acuerdo con el método de fraccionamiento de $\mathrm{P}$ propuesto por Hedley et ál. (1982). En resumen y de acuerdo con esta metodología, cada muestra de suelo es sometida a una extracción secuencial con diferentes reactivos o métodos de extracción, que disuelven en forma selectiva (según el extractante utilizado), las diferentes formas de $\mathrm{P}$ que incluyen primeramente las lábiles y luego las que están ligadas a ciertos componentes, óxidos e hidróxidos de $\mathrm{Fe} / \mathrm{Al}$, Calcio y la fracción orgánica (Henríquez y Killorn 2005).

De esta forma, cada muestra fue sometida a la extracción en forma secuencial, primeramente con resinas de intercambio y luego con $\mathrm{NaHCO}_{3} 0,5 \mathrm{M}$ para obtener con la suma de ambas extracciones el $\mathrm{P}$ inorgánico lábil; seguidamente se utilizó una extracción con $\mathrm{NaOH}$ $0.1 \mathrm{M}$ para determinar el $\mathrm{P}$ ligado a sesquióxidos $\mathrm{Fe} / \mathrm{Al}$, luego una extracción con HCL 0,5M para obtener el $\mathrm{P}$ ligado al calcio y finalmente se realizó una digestión con una mezcla de $\mathrm{H}_{2} \mathrm{SO}_{4}+\mathrm{H}_{2} \mathrm{O}_{2}$ concentrados para determinar el $\mathrm{P}$ residual $\mathrm{u}$ ocluido. El P ligado a la fracción orgánica fue determinado a partir de la diferencia entre el $\mathrm{P}$ determinado a partir de la digestión con $\mathrm{H}_{2} \mathrm{SO}_{4}$ concentrado de alícuotas de los extractos obtenidos de $\mathrm{NaHCO}_{3} 0,5 \mathrm{M}$ y de $\mathrm{NaOH} 0,1 \mathrm{M}$, a la cual se le restó seguidamente la cantidad correspondiente al $\mathrm{P}$ lábil determinado previamente a partir de los mismos extractantes (que constituye el $\mathrm{P}$ lábil inorgánico). Ésta metodología es presentada con mayor detalle por Henríquez y Killorn (2005) en un estudio previo que se realizó bajo condiciones de invernadero.

Seguidamente, los datos obtenidos de las cantidades de $\mathrm{P}$ en las diferentes fracciones o formas del elemento y para cada uso de suelo, fueron analizados estadísticamente con el procedimiento "GLM" y en aquellos casos donde la varianza fue muy alta, se aplicó el procedimiento "PROC MIXED”. La separación de medias se realizó con la prueba Least Significant Difference (LSD) (SAS institute 1996).

\section{RESULTADOS}

A pesar de tener un origen similar, los Andisoles presentan como grupo a nivel mundial, una gran variabilidad en la disponibilidad 
natural de nutrientes en el suelo, esto debido principalmente a la diversidad en los tipos de cenizas y materiales magmáticos de cada zona de influencia volcánica; pese a ello, el $\mathrm{P}$ presenta naturalmente una condición de alta fijación debida a la presencia de alofana (Bertsch et ál. 2000, Alvarado et ál. 2001).

Adicionalmente a este hecho y debido a la variabilidad espacial de las características del suelo, se sabe que aún en áreas relativamente homogéneas por tipo de suelo, esta fertilidad intrínseca (incluyendo el elemento P) así como otras propiedades del suelo, pueden ser modificadas en el tiempo debido a la aplicación de ciertas prácticas de manejo agrícola, como por ejemplo la fertilización, el encalado y el manejo de rastrojos (Bertsch et ál. 2002, Henríquez et ál. 2005).

En el Cuadro 2 se presentan los contenidos obtenidos de las diversas formas de $\mathrm{P}$ determinadas a partir de las muestras de suelo de los 3 lotes muestreados en este estudio; como se mencionó, al ser lotes adyacentes, se partió de la hipótesis inicial que, las posibles diferencias encontradas serían debidas principalmente al uso y manejo del suelo y no a causas pedogenéticas propiamente.

Como se observa, en promedio el P-total en los 2 manejos agrícolas que fueron caña de azúcar y café, fueron estadísticamente similares

Cuadro 2. Formas de P obtenidas por la metodología de fraccionamiento propuesta por Hedley et ál. (1982) en 3 lotes en un Typic Hapludand. Juan Viñas, Costa Rica.

\begin{tabular}{|c|c|c|c|c|c|}
\hline \multirow[t]{2}{*}{ Formas de $\mathrm{P}$} & & Caña de Azúcar & Café & Bosque & Significancia \\
\hline & \multicolumn{5}{|c|}{ mg.kg-1 } \\
\hline \multirow{3}{*}{ Pi-lábil + } & Media & $6,11 \mathbf{b}$ & $35,71 \mathbf{a}$ & $10,32 \mathbf{b}$ & $* *$ \\
\hline & Ámbito & $0,86-13,11$ & $2,35-113,35$ & $2,46-28,84$ & \\
\hline & Des. Std. & 4,24 & 33,96 & 7,50 & \\
\hline \multirow{3}{*}{$\mathrm{P}-\mathrm{SxFeAl}$} & Media & $289,48 \mathbf{b}$ & $442,99 \mathbf{a}$ & $298,45 \mathbf{b}$ & $* *$ \\
\hline & Ámbito & $101,67-555,41$ & $139,50-746,73$ & $109,97-522,12$ & \\
\hline & Des. Std. & 159,17 & 189,63 & 147,92 & \\
\hline \multirow{3}{*}{$\mathrm{P}-\mathrm{Ca}$} & Media & $158,23 \mathbf{a}$ & $53,03 \mathbf{b}$ & $88,66 \mathbf{b}$ & $* *$ \\
\hline & Ámbito & $65,78-303,84$ & $5,52-140,33$ & $14,17-275,00$ & \\
\hline & Des. Std. & 64,18 & 36,96 & 75,78 & \\
\hline \multirow{3}{*}{ P-orgánico + } & Media & $555,31 \mathbf{a}$ & $522,21 \mathbf{a}$ & $385,77 \mathbf{b}$ & ** \\
\hline & Ámbito & $430,94-756,92$ & $289,81-738,37$ & $201,54-629,75$ & \\
\hline & Des. Std. & 93,34 & 133,68 & 128,99 & \\
\hline \multirow{3}{*}{ P-residual } & Media & $907,28 \mathbf{a}$ & $700,16 \mathbf{b}$ & $636,01 \mathbf{b}$ & $* *$ \\
\hline & Ámbito & $728,99-1075,90$ & $426,31-843,74$ & $420,14-856,82$ & \\
\hline & Des. Std. & 99,32 & 112,66 & 146,32 & \\
\hline \multirow{3}{*}{ P-Total } & Media & $1934,74 \mathbf{a}$ & $1754,19 \mathbf{a}$ & $1419,16 \mathbf{b}$ & $* *$ \\
\hline & Ámbito & $1558,70-2516,15$ & $936,86-2269,15$ & $992,88-1825,78$ & \\
\hline & Des. Std. & 296,53 & 339,57 & 271,38 & \\
\hline
\end{tabular}

+Proc Mixed fue aplicado en lugar de GLM debido a la diferencia en la varianza. Con el resto se aplicó el proceso GLM. $* *$ Significancia $\mathrm{p}<0,01$.

Nota: Las filas con las mismas letras son iguales de acuerdo con la prueba de separación de medias LSD a p $<0,05$. 
(1934 y $1754 \mathrm{mg} \cdot \mathrm{kg}^{-1}$ respectivamente), y estas 2 fueron diferentes con respecto al área de bosque (1419 mg. $\left.\mathrm{kg}^{-1}\right)$; en promedio se encontró una diferencia de al menos 400 mg.kg ${ }^{-1}$ de $\mathrm{P}$ entre los manejos de cultivos y de bosque en el transcurso de al menos 20 años, lo cual puede atribuirse a la fertilización y la alta remanencia del elemento en el suelo por los procesos de sorción. Estos valores son considerados altos comparativamente a otros estudios (Beck y Sánchez 1994, Asmak et ál. 2015).

Como se puede observar en el Cuadro 2, el cultivo de café mostró por otro lado los mayores valores de P-lábil $\left(35,71 \mathrm{mg} \cdot \mathrm{kg}^{-1}\right)$ con respecto a caña de azúcar y el bosque (6,11 y $10,32 \mathrm{mg} \cdot \mathrm{kg}^{-1}$ respectivamente); esto concuerda con la mayor frecuencia en la fertilización que se realizan en este cultivo, en comparación a la caña y con mayor razón con respecto al bosque secundario. La fertilización hace que se mantenga la fracción lábil en niveles altos (Bertsch et ál. 2002). Es posible decir que en cultivos de menor frecuencia en la aplicación de fertilizantes, como es el caso de la caña de azúcar, es esperable encontrar en Andisoles, valores de P-lábil menores debidos principalmente a su alta sorción (Ball et ál. 1993, Henríquez et ál. 2004). Los valores porcentuales del P-lábil con respecto al $\mathrm{P}$ total, variaron entre 0,30 a $1,84 \%$ del P total (Cuadro 3), lo cual concuerda con valores reportados por otros autores (Beck y Sánchez 1994, Soto 1998, Neufeldt et ál. 2000, Henríquez y Killorn 2005). En otros estudios y bajo condiciones muy específicas, algunos autores reportan por otro lado que el P-lábil correspondió a valores cercanos a $10 \%$ del P-total (Schrijver et ál. 2012, Borda et ál. 2014).

A diferencia de lo que se esperaba, los manejos agrícolas caña y café, presentaron los mayores contenidos de P-orgánico en comparación al bosque 555, 522 y $385 \mathrm{mg} \cdot \mathrm{kg}^{-1}$ respectivamente (Cuadro 2). De hecho como se observa en el Cuadro 1, el cultivo de la caña presentó el mayor valor de materia orgánica. Pese a lo anterior, esto correspondió a pocas diferencias cuando se analizó la participación de la fracción orgánica del $\mathrm{P}$ en términos porcentuales (Cuadro 3); esta fracción correspondió a 28, 29 y $27 \%$ de participación de esta fracción con respecto al P-total, en la caña, café y bosque, respectivamente. Meason et ál. (2009) mencionan que el efecto de la fertilización en bosques tropicales manejados, fue más evidente en las fracciones inorgánicas que en la fracción orgánica del P. Se considera que valores mayores de un $25 \%$ del $\mathrm{P}$ total constituidos en la fracción orgánica constituyen un buen reservorio de $\mathrm{P}$ en el suelo en comparación a otros estudios (Beck y Sánchez 1994).

Por otro lado el P-residual constituyó la fracción más grande en los 3 tipos de manejo (Cuadros 2 y 3); se encontraron los mayores valores de esta fracción, precisamente en los suelos de caña y café (907 y 700 mg.kg-1 respectivamente) en comparación al bosque (636 mg. $\left.\mathrm{kg}^{-1}\right)$, pese a ello, estos últimos 2 fueron estadísticamente iguales según el análisis realizado (Cuadro 2). En términos porcentuales, esta fracción constituyó entre el 39 al 46\% del P total determinado (Cuadro 3) y se presentó como la mayor.

Cuadro 3. Cantidades relativas de las formas de P obtenidas por la metodología propuesta por Hedley et ál. (1982) en un Typic Hapludand.

\begin{tabular}{lccccc}
\hline Áreas & P-lábil & P-SxFeAl & P-Ca & P-orgánico & P-residual \\
\cline { 2 - 5 } & & & & & 4,18 \\
Caña de Azúcar & 0,32 & 14,96 & 3,02 & 28,70 & 46,89 \\
Café & 2,03 & 25,25 & 6,25 & 27,18 & 39,91 \\
Bosque & 0,72 & 21,03 & 44,82 \\
\hline
\end{tabular}


El análisis de correlación realizado mostró que existe una buena relación entre la fracción lábil y la relacionada a los óxidos e hidróxidos de Fe/Al (0,71 significativo a $\mathrm{p}<0,05$ ) (Cuadro 4). Esto parece sugerir la importancia de ésta fracción con los procesos de sorción ya que pudiese tener relación con la posible ruta del $\mathrm{P}$ aportado por los fertilizantes, lo cual lo convierte en formas de $\mathrm{P}$ no disponibles; esto mismo fue sugerido por Henríquez y Killorn (2005) en un estudio bajo condiciones de invernadero.

Cuadro 4. Coeficientes de correlación de Pearson entre las diferentes formas de P extraídas según la metodología de Hedley (1982) (solo los coeficientes con significancia $\mathrm{p}<0,05$ son presentados), $\mathrm{n}=48$.

\begin{tabular}{lccccc}
\hline Extractant & P-SxFeAl & P-Ca & P-orgánico & P-residual & P-total \\
\hline P-lábil+ & 0,71 & ns & ns & ns & 0,43 \\
P-SxFeAl & - & $n s$ & $n s$ & $n s$ & 0,59 \\
P-Ca & & - & $n s$ & 0,52 & 0,44 \\
P-orgánico++ & & & - & 0,43 & 0,66 \\
P-residual & & & & - & 0,75 \\
\hline
\end{tabular}

+ Sum of resin-Pi and $\mathrm{NaHCO}_{3}$-Pi.

++ Sum of $\mathrm{NaHCO}_{3}-\mathrm{Po}$ and $\mathrm{NaOH}-\mathrm{Po}$.

Meason et ál. (2009) por otro lado, mencionan que los altos contenidos de materia orgánica (típicos en los Andisoles), pueden enmascarar los puntos de sorción del P relacionada a Fe y Al y que la fracción P-Ca, puede ser una fuente importante para el P-lábil.

Se concluye que el método de fraccionamiento y caracterización de las formas de $\mathrm{P}$ en el suelo, podría ayudar a dilucidar el impacto de diferentes prácticas agrícolas sobre este recurso y ampliar el criterio sobre el estado del elemento en el suelo. La técnica de fraccionamiento de $\mathrm{P}$ se muestra como una metodología de diagnostico y monitoreo en el tiempo del elemento. Algunas otras temáticas como por ejemplo, la respuesta de los cultivos a la fertilización fosfórica, estudios de cinética de $\mathrm{P}$ en el suelo, actividad microbial relacionada a $\mathrm{P}$, entre otros, podrían también ser abordados en complemento con los estudios de fraccionamiento de $\mathrm{P}$ en el suelo.

\section{AGRADECIMIENTOS}

El autor desea expresar su agradecimiento a la empresa Hacienda Juan Viñas por facilitar la ejecución del estudio. Asimismo al IPNI (en su momento llamado Instituto de la Potasa y el Fosfato o INPOFOS) por proveer parte del financiamiento para el desarrollo del mismo. Al Dr. Randy Killorn, profesor retirado de Iowa State University por su colaboración y apoyo.

\section{LITERATURA CITADA}

ALVARADO A., BERTSCH F., BORNEMISZA E., CABALCETA G., FORSYTHE W., HENRÍQUEZ C., MATA R., MOLINA E., SALAS R. 2001. Suelos derivados de cenizas volcánicas (Andisoles) de Costa Rica. Asociación Costarricense de la Ciencia del Suelo ACCS. San José, Costa Rica. 111 p.

ASMAK M., HELUF G., MARKKU Y., BIRRU Y. 2015. Phosphorus status, inorganic phosphorus forms, and other physicochemical properties of acid soils of Farta district, Northwestern highlands of Ethiopia. Applied and Environmental Soil Science. 2015. ID 788390

BALL B., SALCEDO I., TIESSEN H., STEWART J. 1993. Short- and long-term phosphorus dynamics in a fertilized Ultisol under sugarcane. Soil Sci. Soc. Am. J. 57:1027-1034.

BECK M., SÁNCHEZ P. 1994. Soil phosphorus fraction dynamics during 18 years of cultivation on a Typic Paleoudult. Soil Sci. Soc. Am. J. 58:1424-1431. 
BERTSCH F., ALVARADO A., HENRÍQUEZ C., MATA R. 2000. Properties, geographic distribution and management of major soil orders of Costa Rica, pp. 265-294. In: C.A.S. Hall, C. Leon and G. Leclerc (eds.). Quantifying sustainable development: The future of tropical economies. Academic Press, San Diego CA.

BERTSCH F., HENRÍQUEZ C., RAMÍREZ F., SANCHO F. 2002. Site-specific nutrient management in the highlands of Cartago province. Better Crops International 16(1):16-19.

BORDA T., CELI L., BÜNEMANN E., OBERSON A., FROSSARD E., BORBERIS E. 2014. Fertilization strategies affect phosphorus forms and release from soils and suspended solids. Journal of Environmental Quality 43(3):10214-10231.

CONDRON L., NEWMAN S. 2011. Revisiting the fundamentals of phosphorus fractionation of sediment and soils. J. Soils Sediments 11:830-840.

FASSBENDER H., BORNEMISZA E. 1987. Química de suelos; con énfasis en suelos de América Latina. $2^{\mathrm{a}}$ Edición. San José, Costa Rica. IICA. 420 p.

FROSSARD E., CONDRON L., OBERSON A., SINAJ S., FARDEU J. 2000. Processes governing phosphorus availability in temperate soils. J. Environ. Qual. 29:15-23.

HEDLEY M., STEWART J., CHAUHAN B. 1982. Changes in inorganic and organic soil phosphorus fractions induced by cultivation practices and by laboratory incubations. Soil Sci. Soc. Am. J. 46:970-976.

HEDLEY M., KIRA G., SANTOS M. 1994. Phosphorus efficiency and the forms of soil phosphorus utilized by upland rice cultivars. Plant and Soil 158:53-62.

HENRÍQUEZ C., BERTSCH F., KILLORN R. 2004. Sugarcane production and changes in soil phosphorus forms after organic and inorganic fertilization. Better Crops 88(2):28-31.

HENRÍQUEZ C. 2005. Sorción y desorción de fósforo en un Andisol de Costa Rica dedicado al cultivo de café, caña de azúcar y bosque. Agronomía Costarricense 29(3):97-105.
HENRÍQUEZ C., KILLORN R. 2005. Soil P forms and $P$ uptake under intensive plant growth in the greenhouse. Agronomía Costarricense 29(2):83-97.

HENRÍQUEZ C., KILLORN R., BERTSCH H. SANCHO F. 2005. La geoestadística en el studio de la variación espacial de la fertilidad de suelos mediante el uso del interpolador Kriging. Agronomía Costarricense 29(2):73-81.

LINQUIST B.A., RUARK M.D., HILL J.E. 2011. Soil order and management practices control soil phosphorus fractions in managed wetlands ecosystems. Nutrient Cycling in Agroecosystems 90(1):51-62.

MAGID J. 1993. Vegetation effects on P fractions in set-aside soils. Plant and Soil 149:111-119.

MEASON D., IDOL T., FRIDAY J., SCOWCROFT P. 2009. Effects of fertilization on phosphorus pools in the volcanic soil of a managed tropical forest. Forest Ecology and Management 256(10):2199-2206.

NEUFELDT H., DA SILVA J., AYARSA M., ZECH W. 2000. Land-use effects on phosphorus fractions in Cerrado oxisols. Biol. Fertil. Soils 31:30-37.

OLSEN S., KHASAWNEH F. 1980. Use and limitations of physical-chemical criteria for assessing the status of phosphorus in soils, pp. 361-410. In: F.E. Khasawneh, E.C. Sample and E.J. Kamprath (ed.). The role of phosphorus in agriculture ASA, CSSA and SSSA. Madison, WI.

SAS INSTITUTE. 1996. SAS/STAT user's guide. Release 6.11. SAS Inst., Cary, NC.

SCHRIJVER A., VESTERDAL L., HANSEN K., DE FRENNE P., AUGSTO L., LUDOVICK D., STALELENS L., DE KEERMAEKER L., DE NEVE S., VERHEYEN K. 2012. Four decades of post-agricultural forest development have caused major redistributions of soil phosphorus fractions. Oecologia 169(1):221-234.

SOTO J.A. 1998. Formas de fósforo y su liberación en andisoles de la región Central Oriental de Costa Rica. Tesis de doctorado, Universidad Superior de Ingenieros Agrónomos y de Montes. España. 155 p.

Todos los derechos reservados. Universidad de Costa Rica. Este artículo se encuentra licenciado con Creative Common Reconocimiento-NoComercial-SinObraDerivada 3.0 Costa Rica.Para mayor información escribir a rac.cia@ucr.ac.cr 
